\title{
Barmer GEK Arztreport 2013 - Fragen an die Experten
}

\section{Nicht die Prävalenz, die Zahl der korrekten Diagnosen ist gestiegen}

\begin{abstract}
? Im Barmer GEK Report 2013 wird über eine massive Zunahme von ADHS-Diagnosen bei Kindern, Jugendlichen und Erwachsenen zwischen 2006 und 2011 berichtet. Für wie zuverlässig halten Sie die aktuellen Zahlen?
\end{abstract}

Professor Claudia Mehler-Wex: ADHS ist eine klar biologisch verankerte Erkrankung, es gibt genetische Hintergründe mit Veränderungen im dopaminergen und noradrenergen Neurotransmittersystem. Dieser Hintergrund hat sich sicherlich nicht geändert, sodass man nicht von erhöhten Prävalenzraten ausgehen darf. Jedoch sind die Versorgungsstrukturen und diagnostischen Möglichkeiten besser und die öffentliche Kenntnis größer geworden, sodass die Zahl derjenigen, die eine ADHS überprüft haben wollen, gestiegen ist und somit auch die Zahl der korrekt gestellten ADHS-Diagnosen. Problematisch sind nicht fachärztlich fundierten Behandlungsversuche mit Stimulan- zien, die zum Teil nicht berechtigt mit einer ADHS-Diagnose verknüpft werden. International geht man von einer ADHS-Prävalenz bei Minderjährigen von 5,3\% aus, insofern liegt die für 2011 festgestellte Diagnoserate von $4,1 \%$, auch wenn sie seit 2006 um etwa $25 \%$ angestiegen ist, noch unter dem Durchschnitt. Bemerkenswert ist allerdings der zum Teil stärker ausgeprägte Schweregrad der ADHS. Hier kommen sicherlich Faktoren wie Reizüberflutung durch Medien, Rückgang des klassischen, schützenden Familiensystems und ein verändertes Erziehungsverhalten zum Tragen.

? Im Report wird aus 6-Jahres-Daten auf eine Lebenszeitprävalenz hochgerechnet. Geben solche Berechnungen ein realistisches Bild?

Mehler-Wex: Da ADHS angeboren ist und die Störung sich ab dem frühem Kindesalter manifestiert, wird die Häufigkeit einer bis

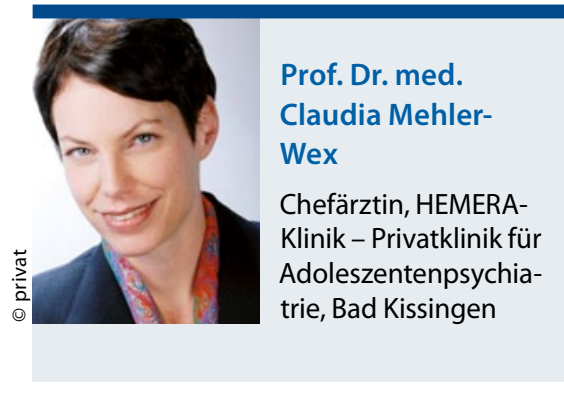

ins Erwachsenenalter persistierenden ADHS eher niedriger eingeschätzt. Durch Reifungsvorgänge im Adoleszentenalter reduziert sich vor allem die Hyperaktivität. Die eingeschränkten Konzentrations- und Aufmerksamkeitsfunktionen sowie Probleme der Verhaltenskontrolle können zum Teil mit bis dahin erworbenen Strategien kompensiert werden, sodass auch die Notwendigkeit einer Stimulanzienbehandlung bei Erwachsenen deutlich sinkt.

\section{Von Erwachsenenpsychiatern inzwischen häufiger erkannt}

? Bei den 20- bis 24-Jährigen hat sich die Zahl der F90-Diagnosen in vier Jahren verdreifacht. Ist die Sensibilität für die Erkrankung bei Erwachsenen gestiegen oder wird oft nicht exakt diagnostiziert?

Professor Wolfgang Retz: Nach den im Barmer GEK Report genannten Zahlen ist die Prävalenz der ADHS in dieser Altersgruppe von $0,27 \%$ auf $0,82 \%$ angestiegen. Um dies richtig zu interpretieren, muss man sich vor Augen führen, dass nach einer epidemiologischen Untersuchung der WHO von einer Prävalenz der Störung im Erwachsenenalter von $3,1 \%$ und einer aktuellen deutschen Studie zufolge sogar von 4,7\% ausgegangen werden muss. Der Anstieg der Diagnosen deutet demnach nicht darauf hin, dass Ärzte bei erwachsenen Patienten ADHS zu häufig und zu unbedacht diagnostizieren. Ich gehe davon aus, dass das in den letzten Jahren angewachsene Wissen über Verlauf und Behandelbarkeit der Störung auch im Erwachsenenalter dazu geführt hat, dass ADHS nun von Erwachsenenpsychiatern häufiger erkannt wird als früher.

? Welche sozialen Probleme kommen angesichts der Hochrechnungen des Reports auf uns zu?

Retz: Die Tatsache dass Betroffene häufig mit Problemen in verschiedenen Lebensfeldern zu kämpfen haben, gehört zu den Kernmerkmalen der Störung. Dass ADHS inzwischen auch bei jungen Erwachsenen offensichtlich häufiger erkannt und diagnostiziert wird, ist ein wichtiger Schritt nach vorne, da nur so adäquate Behandlungsmaßnahmen in die Wege geleitet werden können. Dies ist nicht nur im Hinblick auf die Lebensqualität der Betroffenen, sondern auch unter gesellschaftlicher Gesamtbetrachtung sinnvoll. Denn ADHS geht mit einer Reihe von sozia-

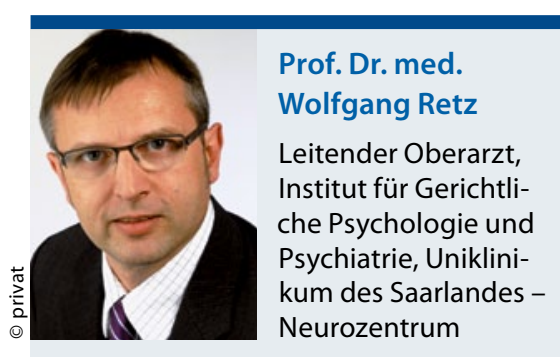

len Problemen einher, die mit Schulschwierigkeiten beginnen, sich in Ausbildung und Berufsleben fortsetzen und vor allem auch Partnerschaften und zwischenmenschliche Beziehungen betreffen können. Auch das Risiko für zivil- und strafrechtliche Probleme und für Übertretungen der Regeln im Straßenverkehr sowie die Zahl der Unfälle jeglicher Art sind bei Personen mit ADHS bereits im Adoleszentenalter erhöht. 